\title{
The broken heart: noninvasive measurement of cardiac autonomic tone
}

\author{
Stephen Oppenheimer
}

\author{
Cerebrovascular Program, The Johns Hopkins Hospital, Meyer 5-185, 600 North Wolfe Street, Baltimore \\ MD 21205, USA
}

Raconteurs relate reports of sudden death under emotional stress, frequently with a retributional message. However, florid embellishments give these tales apochryphal status in serious circles. Recently, interest in this phenomenon has revived. Taggart et al. ${ }^{1}$ demonstrated increased ventricular ectopy and plasma catecholamine levels in stressed normal individuals without heart disease, and the severity of ventricular arrhythmias increases under these circumstances in known cardiac cases. ${ }^{2}$ Catecholamine infusion in humans alters cardiac repolarization and generates arrhythmias. ${ }^{3}$

The demonstration of cardiac chronotropic organization within the rat insular cortex may indicate how emotional stresses alter cardiac autonomic tone. ${ }^{4}$ This area, in humans lying beneath the frontoparietal and temporal opercula, has profuse reciprocal connectivity with the limbic system which is predominantly involved in emotional control. ${ }^{5}$ Prolonged stimulation of cardiac insular sites produces repolarization abnormalities and ventricular ectopy coupled with increased plasma norepinephrine levels and asystolic death. ${ }^{6}$

Shifts in autonomic tone from parasympathetic to sympathetic predominance can induce ventricular ectopy. ${ }^{78}$ Beta-adrenergic blockers reduce ventricular fibrillation incidence in experimental myocardial ischaemia or infarction. ${ }^{9,10}$ Thus, noninvasive assessment of autonomic balance clearly would be useful in patients at risk of sudden cardiac death under emotional circumstances (such as those following myocardial infarction). This can now be achieved by fast Fourier transformation (FFT) of the heart rate (RR interval). This procedure decomposes the envelope of heart rate into its sine wave elements and displays them against their weighted contribution to the overall envelope (power). Using this technique, several peaks of

Correspondence: Stephen Oppenheimer, M.B., M.R.C.P., F.R.C.P.C., F.A.C.P.

Accepted: 28 June 1992 activity have been identified. A low frequency (LF) band $(0.04-0.12 \mathrm{~Hz})$ corresponds to both parasympathetic and sympathetic cardiac influences, whereas a high frequency (HF) band $(0.2-0.28 \mathrm{~Hz})$ corresponds with the respiratory frequency and parasympathetic cardiac tone. ${ }^{11-13}$ In healthy, supine young volunteers, the predominant influence on both peaks is vagal. ${ }^{12}$ On standing, the HF peak decreases markedly, and sympathetic contribution to the LF peak increases. ${ }^{12,13}$ Thus, the relative autonomic contributions are posture dependent in the young. The technique shows good reproducibility on repeat assessments in normal individuals over periods of up to one year. ${ }^{13,14}$

The RR interval FFT is usually analysed using 5-7 minute epochs from Holter data. There are, however, several methodological and practical difficulties. RR variability is a pseudo-random phenomenon yet FFT analysis is mainly applicable to non-random events. Also there is difficulty in determining the relative power of the individual spectral frequencies. To some extent these problems can be overcome by the use of autoregressive algorithms. ${ }^{15,16}$ In addition, FFT analysis assumes a regular heart beat. It is consequently unsuitable for patients in persistent non-sinus rhythms such as atrial fibrillation. Frequent ventricular ectopics also artificially broaden the FFT spectrum. Several methods are available for dealing with this: visual scanning of Holter data and selection of ectopicfree epochs for analysis; the use of a filtering algorithm to eliminate ectopics, ${ }^{14}$ elimination of sharp transients on the tachogram by splining. ${ }^{17}$ The spectrum also depends on the pattern of respiration: regular breathing in response to a metronome signal augments the HF peak compared to spontaneous breathing. ${ }^{13}$

Age also affects the FFT spectrum. Total RR interval FFT spectral content decreases with age as does the area associated with HF and LF peaks. ${ }^{18}$ However, the ratio of $\mathrm{HF}$ to $\mathrm{LF}$ remains unchanged. The effect of standing is also age dependent. The normal LF increase on standing becomes progressively less marked with age. ${ }^{18}$ 
There is also an age-related attenuation of the standing-induced increase in the HF component. However, the LF/HF ratio on standing is unaffected by age. These observations are true during spontaneous breathing. Regular, metronomepaced breathing produces different age-related results. The LF/HF ratio becomes age-dependent both in supine and upright positions. ${ }^{18}$

Thus the balance between parasympathetic and sympathetic effects on the heart is unaffected by age either in the supine or upright position during spontaneous breathing. However, metronome breathing does result in the development of agerelated changes in FFT spectral content during postural manoeuvres.

The HF component in response to metronomeentrained breathing declines linearly until 30 years when no further change is noted whereas the low frequency component shows a linear decline to 62 years. ${ }^{19}$ This implies that respiratory sinus arrhythmia is negligible in both the supine and standing positions after 30 years and suggests a different time course for the changes in sympathetic and parasympathetic influences on the heart.

The effects of medication on the FFT are not entirely clear. Naturally, the spectrum is affected by alpha and beta blockade, but some of the lower frequency components (especially VLF and ULF - see below) may be in part determined by changes in the renin-angiotensin system. ${ }^{11}$ The effects of angiotensin converting enzyme (ACE) inhibitors on the spectrum are not defined as yet.

Stress will shift the balance toward sympathetic predominance emphasizing the LF peak and decreasing the HF peak. ${ }^{20}$ Consequently, patients should be relaxed while heart rate data are being collected.

Spectral power at all frequencies is reduced in severe congestive cardiac failure and is virtually absent at powers greater than $0.04 \mathrm{~Hz}$ in these patients. ${ }^{17}$ This suggests a shift towards sympathetic predominance. Thus, the presence and severity of congestive heart failure must be considered in assessing the spectral result.

FFT analysis of the RR interval has prognostic significance. Bigger et al $^{21}$ divided the frequency domains of the 24 hour FFT of patients 2 weeks after myocardial infarction into four bands: an ultra low frequency (ULF) band of $<0.0033 \mathrm{~Hz}$; a very low frequency (VLF) band 0.0033 to $<0.04 \mathrm{~Hz}$; a LF band 0.04 to $<0.15 \mathrm{~Hz}$; and a HF band of $0.15-0.40 \mathrm{~Hz}$. Decreases in low frequency

\section{References}

1. Taggart, P., Carruthers, M. \& Somerville, W. Electrocardiogram, plasma catecholamines and lipids, and their modification by oxprenolol when speaking before an audience. Lancet 1973, 2: $341-346$. and high frequency power are less predictive of 3 mortality after myocardial infarction. On the other $\stackrel{\mathbb{Q}}{\varrho}$ hand, there is a strong relationship between $C$ decreased ULF, VLF, total power and mortality $\vec{F}$ over the ensuing 2.5 years which is independent of $\stackrel{5}{?}$ other risk factors. Decreased VLF power is? specifically associated with arrhythmic death.

Heart rate variability may be assessed by an $\frac{\bar{\rho}}{\bar{D}}$ alternative technique to FFT. RR intervals can be $\propto$ collected over a 24 hour period, and then displayed $\%$ according to their frequency of occurrence after the $\overrightarrow{0}$ elimination of ectopic beats. This produces a mean and standard deviation for the RR interval. ${ }^{22-25} \vec{\omega}$ This method cannot estimate sympathetic activity, $\frac{\Omega}{\circ}$ but parasympathetic activity can be ascertained by measuring the number of beats whose succeeding. RR intervals are greater than $50 \mathrm{~ms} .{ }^{24}$ Heart rate ${ }_{\infty}$ variability (expressed as the standard deviation ${ }^{\circ}$ around the mean RR interval ascertained over a 24 \% hour period) is reduced with severe coronary artery disease, congestive cardiac failure, and diabetic neuropathy.

Low heart rate variability is a harbinger of $\vec{\nabla}$ sudden death. ${ }^{26,27}$ Decreased heart rate variability $\mathbb{D}_{0}$ is the strongest univariate predictor of mortality $\Phi$ during a 31 month follow-up period after myocar- 믈 dial infarction. ${ }^{14}$ Patients whose heart rate variability is less than 50 milliseconds have $\frac{0}{0} \overrightarrow{0}$ five-fold increase in mortality compared to those with standard deviations greater than 100 millo seconds. This significance of heart rate variability is independent of the mean heart rate, ventricular ectopic activity, ventricular function, and clinical and demographic variables. Cripps, ${ }^{23}$ calculating a heart rate variability index from 24 hour heart rate data, showed that decreased heart rate variability after myocardial infarction was the most potent independent predictor of sudden death and significant ventricular fibrillation.

In conclusion, recent advances in neurophysiology and in cardiology have determined a strong role for the autonomic nervous system in cardiac arrhythmogenesis. The demonstration of cardiac control sites in a brain area involved in integration of emotional and autonomic responses offers a plausible explanation of how emotional events can be associated with sudden death. Tech- $\rightarrow$ niques now exist for noninvasive measurement of autonomic tone at rest and under emotionally $N$ stressful circumstances. These can be used in patients at risk for sudden cardiac death to plan appropriate prophylactic therapy.

2. Lown, B., DeSilva, R.A. \& Lenson, R. Roles of psychologic stress and autonomic nervous system changes in provocation of ventricular premature complexes. Am J Cardiol 1978, 41: 979-985. 
3. Lepeschkin, E., Marchet, H., Schroeder, G., Wagner, R., de Paula e Silva, P. \& Raab, W. Effect of epinephrine and norepinephrine on the electrocardiogram of 100 normal subjects. Am J Cardiol 1960, 5: 594-603.

4. Oppenheimer, S.M. \& Cechetto, D.F. Cardiac chronotropic organisation of the rat insular cortex. Brain Res 1990, 533: 66-72.

5. Yasui, Y., Breder, C.D., Saper, C.B. \& Cechetto, D.F. Autonomic responses and efferent pathways from the insular cortex in the rat. J Comp Neurol 1991, 303: 355-374.

6. Oppenheimer, S.M., Wilson, J.X., Guiraudon, C. \& Cechetto, D.F. Insular cortex stimulation produces lethal cardiac arrhythmias: a mechanism of sudden death? Brain Res 1991, 550: 115-121.

7. Lown, B. \& Verrier, R.L. Neural activity and ventricular fibrillation. $N$ Engl J Med 1976, 294: 1165-1170.

8. Magid, N.M., Eckberg, D.L. \& Sprenkle, J.M. Low dose atropine reduces ventricular vulnerability in normal and ischemic hearts. J Clin Invest 1983, 31: 261A.

9. Anderson, J.L., Rodier, H.E. \& Green, L.S. Comparative effects of beta-adrenergic blocking drugs on experimental ventricular fibrillation threshold. Am J Cardiol 1983, 51: 1196-1202.

10. Gang, E.S., Bigger, J.T. \& Uhl, E.W. Effects of timolol and propranolol on inducible sustained ventricular tachyarrhythmia in dogs with subacute myocardial infarction. $A m J$ Cardiol 1984, 53: 273-281.

11. Akselrod, S., Gordon, D., Ubel, F.A., Shannon, D.C., Barger, A.C. \& Cohen, R.J. Power spectrum analysis of heart rate fluctuations: a quantitative probe of beat-to-beat cardiovascular control. Science 1981, 213: 220-222.

12. Pomeranz, B., Macaulay, R.J.B., Caudill, M.A. et al. Assessment of autonomic function in humans by heart rate spectral analysis. Am J Physiol 1985, 248 (Heart Circ Physiol 17): H151-H153.

13. Pagani, M., Lombardi, F., Guzzetti, S. et al. Power spectral analysis of heart rate and arterial pressure variabilities as a marker of sympatho-vagal interaction in man and conscious dog. Circ Res 1986, 59: 178-193.

14. Kleiger, R.E., Bigger, J.T., Bosner, M.S. et al. Stability over time of variables measuring heart rate variability in normal subjects. Am J Cardiol 1991, 68: 626-630.

15. Box, G.E.P. \& Jenkins, G.M. Time Series Analysis: Forecasting and Control. Holden-Day, New York, 1976.
16. Berger, R.D., Akselrod, S., Gordon, D. \& Cohen, R.J. An efficient algorithm for spectral analysis of heart variability. IEEE Trans Biomed Eng 1986, 33: 900-904.

17. Saul, J.P., Arai, Y., Berger, R.D., Lilly, L.S., Colucci, W.S. \& Cohen, R.J. Assessment of autonomic regulation in chronic congestive heart failure by heart rate spectral analysis. Am J Cardiol 1988, 61: 1292-1299.

18. Schwartz, J.B., Gibb, W.J. \& Tran, T. Aging effects on heart rate. J Gerontol 1991, 46: M99-M106.

19. Shannon, D.C., Carley, D.W. \& Benson, H. Aging of modulation of heart rate. Am J Physiol 1987, 253 (Heart Circ Physiol 22): H874-H877.

20. Pagani, M., Mazzuero, G., Ferrari, A. et al. Sympathovagal interaction during mental stress: a study using spectral analysis of heart rate variability in healthy control subjects and patients with a prior myocardial infarction. Circulation 1991, 83 (Suppl II): 43-51.

21. Bigger, J.T., Fleiss, J.L., Steinman, R.C., Rolnitzky, L.M., Kleiger, R.E. \& Rottman, J.N. Frequency domain measures of heart period variability and mortality after myocardial infarction. Circulation 1992, 85: 164-171.

22. Ewing, D.J., Neilson, J.M.M., Shapiro, C.M., Stewart, J.A. \& Reid, W. Twenty four hour heart rate variability: effects of posture, sleep, and time of day in healthy controls and comparison with bedside tests of autonomic function in diabetic patients. Br Heart J 1991, 65: 239-244.

23. Cripps, T.R., Malik, M., Farrell, T.G. \& Camm, A.J. Prognostic value of reduced heart rate variability after myocardial infarction: clinical evaluation of a new analysis method. Br Heart J 1991, 65: 14-19.

24. Ewing, D.J., Neilson, J.M.M. \& Travis, P. New method for assessing cardiac parasympathetic activity using 24 hour electrocardiograms. Br Heart J 1984, 52: 396-402.

25. Molgaard, H., Sorensen, K.E. \& Bjerregaard, P. Circadian variation and influence of risk factors on heart rate variability in healthy subjects. Am J Cardiol 1991, 68: 777-784.

26. Martin, G.J.M., Magid, N.M., Myers, G., Barnett, P.S. et al. Heart rate variability and sudden death secondary to coronary artery disease during ambulatory electrocardiographic monitoring. Am J Cardiol 1987, 60: 86-89.

27. Kannel, W.B., Kannel, C., Paffenberger, R.S., Cupples, P.H.A. \& Cupples, L. Heart rate and cardiovascular mortality. Am Heart J 1987, 113: 1489-1494. 